\title{
Semisimple-direct-injective modules
}

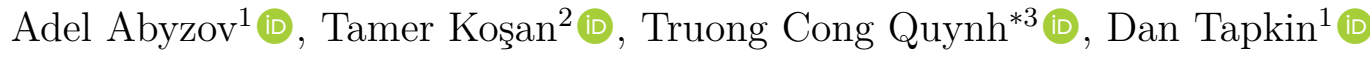 \\ ${ }^{1}$ Department of Algebra and Mathematical Logic, Kazan (Volga Region) Federal University, 18 \\ Kremlyovskaya str., Kazan, 420008 Russia \\ ${ }^{2}$ Department of Mathematics, Faculty of Sciences, Gazi University, Ankara, Turkey \\ ${ }^{3}$ Department of Mathematics, The University of Danang - University of Science and Education, DaNang \\ city, Vietnam
}

\begin{abstract}
The notion of simple-direct-injective modules which are a generalization of injective modules unifies $C 2$ and $C 3$-modules. In the present paper, we introduce the notion of the semisimple-direct-injective module which gives a unified viewpoint of $C 2, C 3$, SSP properties and simple-direct-injective modules. It is proved that a $\operatorname{ring} R$ is Artinian serial with the Jacobson radical square zero if and only if every semisimple-direct-injective right $R$ module has the SSP and, for any family of simple injective right $R$-modules $\left\{S_{i}\right\}_{\mathcal{J}}, \oplus_{\mathcal{J}} S_{i}$ is injective. We also show that $R$ is a right Noetherian right V-ring if and only if every right $R$-module has a semisimple-direct-injective envelope if and only if every right $R$-module has a semisimple-direct-injective cover.
\end{abstract}

Mathematics Subject Classification (2020). 16D50, 16E50

Keywords. $C 2$-module, $C 3$-module, SSP, Artinian serial ring, V-ring, simple-direct-injective module

\section{Introduction}

Throughout this article, unless otherwise stated, all rings have unity and all modules are unital. A right $R$-module $M$ is called

a $C 1$-module provided that every submodule of $M$ is essential in a direct summand of $M$;

a $C 2$-module (or direct-injective) provided that $A$ is a direct summand in $M$ whenever $A$ is a submodule of $M$ such that $A$ is isomorphic to a direct summand in $M$ and

a $C 3$-module if $A$ and $B$ are direct summands in $M$ and $A \cap B=0$, then $A+B$ is a direct summand in $M$.

It is easy to see that each $C 2$-module is also a $C 3$-module. Conversely, for each module $M$, if $M \oplus M$ is a $C 3$-module, then $M$ is a $C 2$-module (see also [1, Corollary 2.6]). However, $C 3$ is a weaker property in general: if $R$ is any integral domain which is not a field, then $R$ is $C 3$, but not $C 2$. Recently, the classes of $C i$-modules $(i=1,2,3)$ are studied and generalizations of them are considered $([1,5,6,12,14])$.

*Corresponding Author.

Email addresses: Adel.Abyzov@kpfu.ru (A. Abyzov), mtamerkosan@gazi.edu.tr (T. Koşan), tcquynh@ued.udn.vn (T.C. Quynh), danil.tapkin@yandex.ru (D. Tapkin)

Received: 02.05.2020; Accepted: 05.09.2020 
We recall also that a module $M$ has the summand sum property (SSP) if the sum of two direct summands is a direct summand of $M$ ([10] and [17]). Clearly, modules having (SSP) are $C 3$.

Recently, Camillo, Ibrahim, Yousif and Zhou [5] obtained that every simple submodule which is isomorphic to a direct summand is itself a direct summand if and only if the sum of any two simple direct summands with zero intersection is again a direct summand [5, Proposition 2.1]. Such modules are called simple-direct-injective (see also [12]). In the present paper, we introduce the concept of semisimple-direct-injective modules. A module is called semisimple-direct-injective if every semisimple submodule isomorphic to a summand is itself a summand, or equivalently if the sum of any two semisimple summands (with zero intersection) is again a summand (see Proposition 2.1). Theorem 3.4 in [5] addressed the question of when every simple-direct-injective module is $C 3$, and they proved that every simple-direct-injective right $R$-module is $C 3$ if and only if $R$ is an Artinian serial ring with Jacobson radical square zero. In Theorem 2.10, we prove that $R$ is an Artinian serial ring with Jacobson radical square zero if and only if every semisimple-direct-injective right $R$-module has the SSP and $\oplus_{\mathfrak{j}} S_{i}$ is injective for any family of simple injective modules $\left\{S_{i}\right\}_{\text {J }}$.

Enochs [7] introduced the notation of injective cover as the dual notation of the injective envelope, and proved that a ring $R$ is right Noetherian if and only if every right $R$-module has an injective cover. In Section 3, we are concerned with semisimple-direct-injective envelopes and covers, namely sdi-envelopes and sdi-covers. In Theorem 3.4, it is shown that the classes of semisimple-direct-injective modules over a ring $R$ provide for sdi-envelopes and sdi-covers only if $R$ is a right Noetherian V-ring.

A ring is called a right $V$-ring if every simple right $R$-module is injective. In Section 4 , we study some natural connections between V-rings and semisimple-direct-injective modules which are similar to simple-direct-injective modules. For instance, we obtain that a ring is right Noetherian and a right V-ring if and only if every right $R$-module is semisimpledirect-injective if and only if every direct sum of two semisimple-direct-injective modules is semisimple-direct-injective (Theorem 2.11).

Throughout this article, a submodule $N$ of an $R$-module $M$ is called essential in $M$, denoted by $N \leq_{e} M$, if for any nonzero submodule $L$ of $M, L \cap M \neq 0$. We write $J(R)$ and $\operatorname{Soc}\left(R_{R}\right)$ for the Jacobson radical and the socle of $R$, respectively. We also write $N \leq_{d} M$ and $E(M)$ to indicate that $N$ is a direct summand of $M$ and the injective envelope of $M$, respectively. For a nonempty subset $X$ of a ring $R$, the left annihilator of $X$ in $R$ is $l(X)=\{r \in R: r x=0$ for all $x \in X\}$. For any $a \in R$, we write $l(a)$ for $l(\{a\})$. Right annihilators are defined similarly. General background material can be found in [3], [6], [13] and [18].

\section{Semisimple-direct-injective modules}

Proposition 2.1. The followings are equivalent for a right $R$-module $M$.

(1) For any semisimple submodules $A, B$ of $M$ with $A \cong B \leq_{d} M, A$ is a summand of $M$.

(2) For any semisimple summands $A, B$ of $M$ with $A \cap B=0$, the sum $A \oplus B$ is a summand of $M$.

(3) For any semisimple summands $A, B$ of $M, A+B \leq_{d} M$.

(4) If $M=A_{1} \oplus A_{2}$ with $A_{1}$ semisimple and $f: A_{1} \rightarrow A_{2}$ is a homomorphism, then $\operatorname{Im}(f) \leq_{d} A_{2}$.

Proof. (1) $\Rightarrow(2)$ Assume $M=A \oplus A^{\prime}$ and let $\pi: A \oplus A^{\prime} \rightarrow A^{\prime}$ be the canonical projection. Then $A \oplus B=A \oplus \pi(B)$ is a direct summand of $M$ as $\pi(B) \cong B$.

$(2) \Rightarrow(3)$ Straightforward. 
$(3) \Rightarrow(4)$ Let $X:=\left\{a-f(a): a \in A_{1}\right\}$. Clearly, $X \oplus A_{2}=M$. Furthermore, $A_{1} \oplus \operatorname{Im}(f)=A_{1}+X$ which is a direct summand of $M$ by the hypothesis. Now, the conclusion follows.

(4) $\Rightarrow(1)$ Let $B \oplus B^{\prime}=M$ and $\theta: B \rightarrow A$ be an isomorphism. Also set $f:=\pi_{\left.\right|_{A}} \theta$, where $\pi: B \oplus B^{\prime} \rightarrow B^{\prime}$ is the canonical projection. Then $\operatorname{Im}(f)=\pi(A) \leq_{d} A_{2}$ by the assumption, so that $B+A=B \oplus \pi(A) \leq_{d} M$. Since $A \leq_{d} A+B$, we get $A \leq_{d} M$ as well.

A module $M$ is called semisimple-direct-injective if $M$ satisfies the equivalent conditions of Proposition 2.1. A ring $R$ is called right semisimple-direct-injective if $R_{R}$ is semisimpledirect-injective.

Example 2.2. Every indecomposable module is semisimple-direct-injective. In particular, $\mathbb{Z}_{\mathbb{Z}}$ is a semisimple-direct-injective module which is not direct-injective.

Example 2.3. Every semisimple-direct-injective module is simple-direct-injective. The converse is true if the module is finitely generated or it has ACC on summands by [5, Proposition 2.5] and [5, Corolllary 2.9], respectively.

Proposition 2.4. If any semisimple summand of a right $R$-module $M$ is invariant under all idempotents of $\operatorname{End}(M)$, then $M$ is semisimple-direct-injective.

Proof. Let $A, B$ be semisimple summands of the module $M$ with $A \cap B=0$. Let $M=A \oplus$ $A^{\prime}$ for some submodule $A^{\prime}$ of $M$. Consider the projections $\pi_{1}: M \rightarrow A$ and $\pi_{2}: M \rightarrow A^{\prime}$. Since $B$ is invariant under all idempotents of $\operatorname{End}(M)$, we obtain

$$
\begin{aligned}
B & \leq \pi_{1}(B) \oplus \pi_{2}(B) \\
& \leq\left[\pi_{1}(M) \cap B\right] \oplus\left[\pi_{2}(M) \cap B\right] \\
& =(A \cap B) \oplus\left(A^{\prime} \cap B\right) \\
& =A^{\prime} \cap B \leq A^{\prime}
\end{aligned}
$$

This follows that $B$ is a direct summand of $M$ and so $A^{\prime}=B \oplus B^{\prime}$ for some submodule $B^{\prime}$ of $A^{\prime}$. Thus,

$$
M=A \oplus A^{\prime}=A \oplus\left(B \oplus B^{\prime}\right)=(A \oplus B) \oplus B^{\prime} .
$$

Recall that $R$ is called a right $V$-ring if every simple right $R$-module is injective. By Theorem 2.11 below, a ring $R$ is right Noetherian and a right V-ring if and only if every right $R$-module is semisimple-direct-injective. On the other hand, a ring $R$ is a right $\mathrm{V}$-ring if and only if every right $R$-module is simple-direct-injective by [5, Proposition 4.1].

Example 2.5. (i) Let $Q:=\prod_{i=1}^{\infty} F_{i}$ with $F_{i}:=\mathbb{Z}_{2}$ and $R$ be the subring of $Q$ generated by $\bigoplus_{i=1}^{\infty} F_{i}$ and $1_{Q}$. Then $R$ is a commutative, non self-injective V-ring and $\operatorname{Soc}(R)$ is essential in $R$. We deduce that $R$ is not Noetherian. Thus one infers that there exists a simple-direct-injective module over $R$ which is not semisimple-direct-injective.

(ii) Let $V$ be an infinite-dimensional vector space over $F$. Let $Q:=\operatorname{End}_{F}(V), J:=$ $\left\{x \in Q: \operatorname{dim}_{F}(x V)<+\infty\right\}$ and $R:=F+J$. Then $R$ is a right V-ring (see [9, Example 6.19]) and $R$ is not right Noetherian. Similarly (i), there is a simple-direct-injective right $R$-module which is not semisimple-direct-injective.

Example 2.6. If $M$ is an indecomposable right $R$-module which is not simple, then $M \oplus E(M)$ is a semisimple-direct-injective module. Indeed, by [5, Lemma 3.3], $M \oplus E(M)$ has no simple summands. 
Example 2.7. Given a field $F$ and an isomorphism $F \rightarrow \bar{F} \subseteq F$ defined by $a \mapsto \bar{a}$, let $R$ be the right $F$-space on basis $\{1, t\}$ with multiplication given by $t^{2}=0$ and $a t=t \bar{a}$ for all $a \in F$. Assume that $1<\operatorname{dim}_{\bar{F}}(F)<\infty$. By Example 2.6, $R \oplus E(R)$ is a semisimpledirect-injective module which is not $C 3$ (has not the SSP) by [5, Example 3.6].

Proposition 2.8. If $M=\oplus_{i \in \mathcal{J}} E_{i}$ is a direct sum of indecomposable injective right $R$ modules $E_{i}$, then $M$ is a semisimple-direct-injective module.

Proof. Let $A$ be the sum of the simples $E_{i}$ and $B$ be the sum of the non-simple ones. If $S$ is isomorphic to a semisimple direct summand of $M$, then all simple summands of $S$ are clearly injective, so that $S \cap B=0$. Since $(B \oplus S) \cap A$ is a direct summand of $A$, we get the former is a direct summand of $M$, whence $S$ is a direct summand of $M$.

Corollary 2.9. Let $\left\{S_{i}\right\}_{\mathcal{J}}$ be a family of simple injective modules and $\left\{E\left(S_{j}\right)\right\}_{\mathcal{K}}$ be a family of injective envelopes of simple non-injective modules $S_{j}$. Then $M=\left(\oplus_{i \in \mathfrak{J}} S_{i}\right) \oplus$ $\left(\oplus_{j \in \mathcal{K}} E\left(S_{j}\right)\right)$ is a semisimple-direct-injective module.

A module is uniserial if the lattice of its submodules is totally ordered under inclusion. A ring $R$ is called right uniserial if $R_{R}$ is a uniserial module. A ring $R$ is called serial if both modules ${ }_{R} R$ and $R_{R}$ are direct sums of uniserial modules.

Now we investigate when semisimple-direct-injective modules have the SSP.

Theorem 2.10. The followings are equivalent for a ring $R$ :

(1) $R$ is an Artinian serial ring with $J(R)^{2}=0$.

(2) (a) Every semisimple-direct-injective right $R$-module is a C3-module.

(b) For any family of simple injective modules $\left\{S_{i}\right\}_{\mathcal{J}}, \oplus_{\mathfrak{J}} S_{i}$ is injective .

(3) (a) The right socle of $R$ is finitely generated.

(b) Every semisimple-direct-injective right $R$-module is quasi-injective.

Proof. (1) $\Rightarrow(3)$ For any module $M$ over an Artinian serial ring $R$ with $J(R)^{2}=0$, we have a decomposition $M=A \oplus M$, where $A$ is semisimple and $B$ is a sum of injective serial modules of length 2 by $[6,13.5]$. So, it is obvious that semisimple-direct-injective right $R$-modules are precisely those with $A$ orthogonal to $B$. In this case, $B$ is injective and $A$ is injective relative to $B$. Thus, $M$ is quasi-injective.

$(3) \Rightarrow(2)$ As each quasi-injective module is a C3-module, one only needs to verify $(b)$ : If every semisimple-direct-injective right $R$-module is quasi-injective and every module having the zero socle is a semisimple-direct-injective module, then $R$ is right semi-Artinian (i.e., all nonzero modules have nonzero socle). So, $E\left(R_{R}\right)=E\left(T_{1}\right) \oplus E\left(T_{2}\right) \oplus \cdots \oplus E\left(T_{n}\right)$ where each $T_{i}$ is a minimal right ideal of $R$. Let $\left\{S_{i}\right\}_{\mathbb{N}}$ be a family of simple right $R$ modules. Let $M:=\left(\oplus_{\mathbb{N}} E\left(S_{i}\right)\right) \oplus\left(\oplus_{j=1}^{n} E\left(T_{j}\right)\right)$. By Lemma $2.8, M$ is a semisimple-directinjective module and so, by (3-b), $M$ is a quasi-injective module. Now one infers that $\oplus_{\mathbb{N}} E\left(S_{i}\right)$ is $E\left(R_{R}\right)$-injective and hence it is injective.

$(2) \Rightarrow(1)$ We first prove $R$ is right Noetherian. Let $\left\{S_{i}\right\}_{\mathbb{N}}$ be a family of simple right $R$-modules. We claim that $\oplus_{\mathbb{N}} E\left(S_{i}\right)$ is an injective module. By [4, Theorem 1.3], one infers that there exists an infinite subset $\mathcal{J}$ of $\mathbb{N}$ such that $\oplus_{\mathfrak{J}} E\left(S_{i}\right)$ is injective. Write $\mathbb{N}=\mathcal{J}_{1} \cup \mathcal{J}_{2}$ such that $S_{i}$ is injective if $i \in \mathcal{J}_{1}$ and $S_{j}$ is not injective if $j \in \mathcal{J}_{2}$. By the assumption, $\oplus_{\mathfrak{J}_{1}} S_{i}$ is injective. Now we can assume that $\left|\mathcal{J}_{2}\right|$ is infinite. Note that $M=\left(\oplus_{\mathfrak{I}_{2}} E\left(S_{j}\right)\right) \oplus E\left(\oplus_{\mathfrak{I}_{2}} E\left(S_{j}\right)\right)$ has no simple summands. Hence $M$ is a semisimpledirect-injective module, and so it is a C3-module. So, $\oplus_{\mathfrak{f}_{2}} E\left(S_{j}\right)$ is an injective module. Thus $R$ is right Noetherian. Now, by the same proof of $(1) \Rightarrow(3)$ of Theorem 3.4 in [5], one infers that $R$ is an Artinian serial ring with $J(R)^{2}=0$.

The following observations give some connections between (right Noetherian) right Vrings and semisimple-direct-injective modules. 
Theorem 2.11. The following conditions are equivalent for a ring $R$ :

(1) $R$ is a right Noetherian and right $V$-ring.

(2) Every right $R$-module is semisimple-direct-injective.

(3) Direct sum of two semisimple-direct-injective right $R$-modules is semisimple-directinjective.

Proof. Recall that $R$ is a right Noetherian and right V-ring if and only if every semisimple module is injective.

$(1) \Rightarrow(2),(3)$ are obvious.

(2) $\Rightarrow(1)$ If $A$ is a semisimple right $R$-module, then, by the assumption, $M=A \oplus E(A)$ is a semisimple-direct-injective module. By Proposition 2.1, $A$ is a direct summand of $E(A)$ and hence $A$ is injective. Thus $R$ is a right Noetherian right V-ring.

$(3) \Rightarrow(1)$ is similar to $(2) \Rightarrow(1)$.

Corollary 2.12. $R$ is semisimple Artinian if and only if every semisimple-direct-injective right $R$-module is injective.

Proof. Assume that every semisimple-direct-injective right $R$-module is injective. We deduce that every semisimple right $R$-module is injective. So, $R$ is a right Noetherian right $\mathrm{V}$-ring.

If $R$ is not right semi-Artinian, there exists a non-zero right $R$-module $M$ with $\operatorname{Soc}(M)=$ 0 . Clearly, $M$ and its submodules are injective, a contradiction.

We recall Example 2.3 before the following corollary.

Corollary 2.13. Let $R$ be a right $V$-ring. Then $R$ is right Noetherian if and only if every simple-direct-injective right $R$-module is semisimple-direct-injective.

In [5, Theorem 4.4], authors give a new answer to Fisher's question [8]: When are regular rings right $\mathrm{V}$-rings?. They proved that a regular ring $R$ is a right $\mathrm{V}$-ring if and only if every cyclic right $R$-module is simple-direct-injective. Recall that a ring $R$ is called (von Neumann) regular if for every $a \in R$, there exists some $b \in R$ such that $a=a b a$.

Theorem 2.14. Let $R$ be a regular ring. The following conditions are equivalent:

(1) $R$ is a right $V$-ring.

(2) Every cyclic right $R$-module is semisimple-direct-injective.

(3) Every cyclic right $R$-module is simple-direct-injective.

Proof. This follows from [5, Theorem 4.4] and Example 2.3.

A right $R$-module $M$ is called strongly soc-injective if for any right $R$-module $N$ and any semisimple submodule $K$ of $N$, every $R$-homomorphism $f: K \rightarrow M$ extends to $N$ [2]. By [2, Proposition 16], a right $R$-module $M$ is strongly soc-injective if and only if $M=E \oplus T$, where $E$ is injective and $\operatorname{Soc}(T)=0$. It is easy to see that every strongly soc-injective module is semisimple-direct-injective.

Proposition 2.15. The following conditions are equivalent for a ring $R$ :

(1) $R$ is a right Noetherian right $V$-ring.

(2) Every semisimple-direct-injective module is strongly soc-injective.

Proof. $(1) \Rightarrow(2)$. Let $M$ be a semisimple-direct-injective module. Assume that $\operatorname{Soc}(M)$ is non-zero. Hence, $M$ has a decomposition $M=\operatorname{Soc}(M) \oplus T$ such that $\operatorname{Soc}(M)$ is injective and $\operatorname{Soc}(T)=0$. Thus, $M$ is a strongly soc-injective module.

$(2) \Rightarrow(1)$ Let $M$ be a semisimple module. Then, $M$ is a strongly soc-injective module, write $M=E \oplus T$, where $E$ is injective and $\operatorname{Soc}(T)=0$. Furthermore, we have $T=\operatorname{Soc}(T)$ and so $M=E$ is injective. 
Recall that a right $R$-module $M$ is called mininjective if, for every simple right ideal $K$ of $R$, each $R$-homomorphism $f: K \rightarrow M$ extends to $g: R \rightarrow M$; that is, $f=m$. is multiplication by some $m \in M([14])$.

Lemma 2.16 ([14, Theorem 2.36]). The following conditions are equivalent for a ring $R$ :

(1) Every right $R$-module is mininjective.

(2) Every cyclic right $R$-module is mininjective.

(3) $K^{2} \neq 0$ for every simple right ideal $K$ of $R$.

(4) $\operatorname{Soc}\left(R_{R}\right) \cap J(R)=0$.

(5) $R$ is right mininjective and $\operatorname{Soc}\left(R_{R}\right)$ is projective as a right $R$-module.

A ring $R$ is called right universally mininjective if it satisfies the conditions in Lemma 2.16 .

Lemma 2.17. The following conditions are equivalent for a ring $R$ :

(1) $R$ is right universally mininjective.

(2) $R$ is right semisimple-direct-injective and every minimal right ideal of $R$ is projective as a right $R$-module.

Proof. (1) $\Rightarrow(2)$. Assume that $R$ is right universally mininjective. Then, every minimal right ideal of $R$ is a direct summand of $R_{R}$ by Lemma 2.16. It follows that $R$ is a right simple-direct-injective ring, and so it is semisimple-direct-injective.

$(2) \Rightarrow(1)$. We show that $R$ is right mininjective. Indeed, let $K$ be a minimal right ideal of $R$. Then, $K$ is a projective module, and so $K$ is isomorphic to a direct summand of $R_{R}$. We have that $R$ is right semisimple-direct-injective and obtain that $K$ is a direct summand of $R_{R}$. We deduce that $R$ is right mininjective. Thus, $R$ is right universally mininjective by Lemma 2.16.

Theorem 2.18. The following conditions are equivalent for a ring $R$ :

(1) $R$ is semisimple Artinian.

(2) $R$ satisfies the following conditions:

(a) $R$ is right semisimple-direct-injective with $\operatorname{Soc}\left(R_{R}\right) \leq_{e} R_{R}$ and projective as a right $R$-module.

(b) Every ascending chain

$$
r\left(a_{1}\right) \subseteq r\left(a_{2} a_{1}\right) \subseteq \cdots
$$

terminates for every infinite sequence $a_{1}, a_{2}, \ldots$ of elements in $R$.

Proof. (1) $\Rightarrow(2)$ This is obvious.

$(2) \Rightarrow(1)$ By $(2-a), R$ is a right universally mininjective ring and $\operatorname{Soc}\left(R_{R}\right) \leq \operatorname{Soc}\left({ }_{R} R\right)$ by Lemma 2.17. Hence $\operatorname{Soc}\left({ }_{R} R\right)$ is essential in $R_{R}$. From [15, Theorem 2.2] we infer that $R$ is a right perfect ring. Furthermore, $\operatorname{Soc}\left(R_{R}\right) \cap J(R)=0$ and $\operatorname{Soc}\left(R_{R}\right) \leq_{e} R_{R}$, which implies that $J(R)=0$. Thus $R$ is a semisimple Artinian ring.

We denote the nil radical $N(R)$ of $R$ by $N(R)=\sum\{I \mid I$ is nil right ideal of $R\}$.

Corollary 2.19. If $N(R)=0, \operatorname{Soc}\left(R_{R}\right) \leq_{e} R_{R}$ and every ascending chain

$$
r\left(a_{1}\right) \subseteq r\left(a_{2} a_{1}\right) \subseteq \cdots
$$

terminates for every infinite sequence $a_{1}, a_{2}, \ldots$ of elements in a ring $R$, then $R$ is a semisimple Artinian ring.

Proof. Let $I$ be an arbitrary minimal right ideal of $R$. From the hypothesis $N(R)=0$ it immediately follows that $I^{2} \neq 0$. Therefore, $I$ is a direct summand of $R_{R}$. It follows that $R$ is right semisimple-direct-injective and every minimal right ideal of $R$ is projective as a right $R$-module. Thus $R$ is a semisimple Artinian ring. 
Corollary 2.20 ([18, 4.3]). A right Artinian ring $R$ with $N(R)=0$ is semisimple Artinian.

We finish this section with the study of the following question:

" Does there exist a right semisimple-direct-injective ring that is not left semisimpledirect-injective?"

Rings of formal triangular matrices also serve as a source of examples of rings with non-symmetrical properties. Below we give an example of a formal triangular matrices ring that answers positively the previous question.

Given the $R$-S-bimodule $M$ we denote

$$
l(M)=\{r \in R \mid r M=0\}, r(M)=\{s \in S \mid M s=0\}
$$

Theorem 2.21. The following conditions are equivalent for a formal triangular matrices $\operatorname{ring} K=\left(\begin{array}{cc}R & M \\ 0 & S\end{array}\right)$

(1) $K$ is a right semisimple-direct-injective ring;

(2) (a) For any semisimple submodules $A, B$ of $l(M)$ with $A \cong B \leq_{d} R_{R}$, $A$ is a summand of $R_{R}$.

(b) For any semisimple submodules $A, B$ of $S_{S}$ with $A \cong B \leq_{d} S_{S}$, $A$ is a summand of $S_{S}$ and $A \leq r(M)$.

Proof. (1) $\Rightarrow(2)$ (a) Let $A$ be a semisimple submodule of $R_{R}, A \cong B \leq_{d} R_{R}$ and $A, B \leq l(M)$. Then, there exists a submodule $B^{\prime}$ of $R_{R}$ such that $R_{R}=B \oplus B^{\prime}$. It follows that there is a decomposition $K_{K}=\left(\begin{array}{cc}B & 0 \\ 0 & 0\end{array}\right) \oplus\left(\begin{array}{cc}B^{\prime} & M \\ 0 & S\end{array}\right)$. We have that an $K$-isomorphism $\left(\begin{array}{cc}A & 0 \\ 0 & 0\end{array}\right) \cong\left(\begin{array}{cc}B & 0 \\ 0 & 0\end{array}\right)$ of $K$-modules and obtain that there exists a submodule $L$ of $K_{K}$ such that we have a decomposition $K_{K}=\left(\begin{array}{cc}A & 0 \\ 0 & 0\end{array}\right) \oplus L$. Let $A^{\prime}=\left\{a \in R \mid \exists m \in M, \exists s \in S:\left(\begin{array}{cc}a & m \\ 0 & s\end{array}\right) \in L\right\}$. One can check that $R_{R}=A \oplus A^{\prime}$.

(b) Let $A$ be a semisimple submodule of $S_{S}, A \cong B \leq_{d} S_{S}$. Using arguments similar to those in the proof of (a), we can show that $A \leq_{d} S_{S}$. Assume that $M A \neq 0$. Then, there exists a simple submodule $A_{0}$ of $A$ such that $M A_{0} \neq 0$. One can check that there is an isomorphism of $K$-modules $\left(\begin{array}{cc}0 & 0 \\ 0 & A_{0}\end{array}\right) \cong\left(\begin{array}{cc}0 & M A_{0} \\ 0 & 0\end{array}\right)$. Since $\left(\begin{array}{cc}0 & 0 \\ 0 & A_{0}\end{array}\right) \leq_{d} K_{K}$, then we get a contradiction with the condition of (1). It follows that $M A=0$ or $A \leq r(M)$.

(2) $\Rightarrow(1)$ Firstly, let $A$ be a simple submodule of $K_{K}, A \cong A^{\prime} \leq_{d} K_{K}$. It follows, from the condition of (2), that either $A^{\prime}=\left(\begin{array}{ll}e & 0 \\ 0 & 0\end{array}\right) K=\left(\begin{array}{cc}e R & 0 \\ 0 & 0\end{array}\right)$, or $A^{\prime}=\left(\begin{array}{cc}0 & 0 \\ 0 & e^{\prime}\end{array}\right) K=$ $\left(\begin{array}{cc}0 & 0 \\ 0 & e^{\prime} S\end{array}\right)$ for some $e^{2}=e \in R$ and $e^{\prime 2}=e^{\prime} \in S$.

Assume that $A^{\prime}=\left(\begin{array}{ll}e & 0 \\ 0 & 0\end{array}\right) K$ and $f: A^{\prime} \rightarrow A$ is an isomorphism of $K$-modules. Since $A^{\prime}\left(\begin{array}{ll}0 & 0 \\ 0 & 1\end{array}\right)=0$, then $S=\left(\begin{array}{cc}A_{0} & 0 \\ 0 & 0\end{array}\right)$, where $A_{0}$ is a simple submodule of $R_{R}$.

Assume that $A^{\prime}=\left(\begin{array}{cc}0 & 0 \\ 0 & e^{\prime}\end{array}\right) K$ and $f: A^{\prime} \rightarrow A$ is an isomorphism of $K$-modules. Since $A^{\prime}\left(\begin{array}{ll}1 & 0 \\ 0 & 0\end{array}\right)=0$ then $f\left(\left(\begin{array}{cc}0 & 0 \\ 0 & e^{\prime}\end{array}\right)\right)=\left(\begin{array}{cc}0 & m \\ 0 & s\end{array}\right)$ with $m \in M, s \in S$. We have $f\left(\left(\begin{array}{cc}0 & 0 \\ 0 & e^{\prime}\end{array}\right)\right)\left(\begin{array}{cc}0 & 0 \\ 0 & e^{\prime}\end{array}\right)=f\left(\left(\begin{array}{cc}0 & 0 \\ 0 & e^{\prime}\end{array}\right)\right)$ and $\operatorname{get}\left(\begin{array}{cc}0 & m \\ 0 & s\end{array}\right)=\left(\begin{array}{cc}0 & m e^{\prime} \\ 0 & s e^{\prime}\end{array}\right)=\left(\begin{array}{cc}0 & 0 \\ 0 & s e^{\prime}\end{array}\right)$. Thus $A=\left(\begin{array}{cc}0 & 0 \\ 0 & B\end{array}\right)$, where $B$ is a simple submodule of $S_{S}$. 
Now, we assume that $A$ is a semisimple submodule of $K_{K}$ and $A \cong B \leq_{d} K_{K}$. It follows, from the above reasoning, that there are submodules $C, C^{\prime}$ of $R_{R}$ and $D, D^{\prime}$ of $S_{S}$ such that $A=\left(\begin{array}{cc}C & 0 \\ 0 & D\end{array}\right)$ and $B=\left(\begin{array}{cc}C^{\prime} & 0 \\ 0 & D^{\prime}\end{array}\right)$. Since $A \cong B$, it is easy to verify that $C_{R} \cong C_{R}^{\prime}$ and $D_{R} \cong D_{R}^{\prime}$. We have that $B \leq_{d} K_{K}$ and obtain that $C^{\prime} \leq_{d} R_{R}$, and $D^{\prime} \leq_{d} S_{S}$. Then, it follows, from the conditions of (2), that there are submodules $E \leq R_{R}, F \leq S_{S}$ such that we have a decomposition $C \oplus E=R_{R}, D \oplus F=S_{S}$. Thus, we have a decomposition $K_{K}=\left(\begin{array}{cc}C & 0 \\ 0 & D\end{array}\right) \oplus\left(\begin{array}{cc}E & M \\ 0 & F\end{array}\right)=A \oplus\left(\begin{array}{cc}E & M \\ 0 & F\end{array}\right)$.

Example 2.22. Let $Q:=\prod_{i=1}^{\infty} F_{i}$ with $F_{i}:=\mathbb{Z}_{2}$ and $R$ be the subring of $Q$ generated by $\bigoplus_{i=1}^{\infty} F_{i}$ and $1_{Q}$. Consider the right action $R$ on $T_{2}\left(\mathbb{Z}_{2}\right)=\left(\begin{array}{cc}\mathbb{Z}_{2} & \mathbb{Z}_{2} \\ 0 & \mathbb{Z}_{2}\end{array}\right)$ which are defined by the relations

$$
\left(\begin{array}{ll}
a & b \\
0 & c
\end{array}\right)\left(\alpha 1_{Q}+\beta\right)=\left(\begin{array}{cc}
a \alpha & b \\
0 & c \alpha
\end{array}\right)
$$

where $\alpha \in \mathbb{Z}_{2}, \beta \in \bigoplus_{i=1}^{\infty} F_{i}$. Then $T_{2}\left(\mathbb{Z}_{2}\right)$ is $T_{2}\left(\mathbb{Z}_{2}\right)$ - $R$-bimodule. Consider the formal triangular matrices ring $K=\left(\begin{array}{cc}T_{2}\left(\mathbb{Z}_{2}\right) & T_{2}\left(\mathbb{Z}_{2}\right) T_{2}\left(\mathbb{Z}_{2}\right)_{R} \\ 0 & R\end{array}\right)$. Since the ring $T_{2}\left(\mathbb{Z}_{2}\right)$ is not left (and right) semisimple-direct-injective, it follows, from the left-sided analogue of Theorem 2.21 , that the ring $K$ is not left semisimple-direct-injective. Since $l\left(T_{T_{2}\left(\mathbb{Z}_{2}\right)} T_{2}\left(\mathbb{Z}_{2}\right)_{R}\right)=0$ and $r\left(T_{2}\left(\mathbb{Z}_{2}\right) T_{2}\left(\mathbb{Z}_{2}\right)_{R}\right)=\operatorname{Soc}(R)$, then conditions (2)(a) and (2)(b) of Theorem 2.21 hold. Thus, the ring $K$ is right semisimple-direct-injective.

\section{Semisimple-direct-injective envelopes and covers}

An $R$-homomorphism $g: E \rightarrow M$ is called a semisimple-direct-injective cover (a $C 3$ cover [1], respectively) for short an sdi-cover, of a right $R$-module $M$ if $E$ is a semisimpledirect-injective module (a $C 3$ module, respectively) such that:

(i) Any diagram

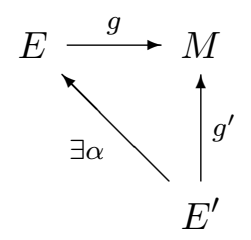

with $E$ a semisimple-direct-injective module (a $C 3$ module, respectively), can be commutatively completed.

(ii) If any endomorphism $\alpha: E \rightarrow E$ satisfies $g \alpha=g$, then $\alpha$ must be an automorphism of $E$.

Dually, the notion of the semisimple-direct-injective envelope can be defined.

Lemma 3.1. Assume that $N$ is a non-injective semisimple module. Then the module $M=N \oplus E(N)$ does not have an sdi-envelope and an sdi-cover.

Proof. Consider the inclusion map (note that, it is the semisimple-direct-injective envelope monomorphism)

$$
\iota: N \oplus E(N) \rightarrow E,
$$

where $E$ is a semisimple-direct-injective module. Since the modules $N$ and $E(N)$ are semisimple-direct-injective, there exist $f_{1}: E \rightarrow N$ and $f_{2}: E \rightarrow E(N)$ such that $f_{i} \iota=\pi_{i}$, where $\pi_{1}: M \rightarrow N$ and $\pi_{2}: M \rightarrow E(N)$ are the projections. Now there exists $f: E \rightarrow$ 
$N \oplus E(N)$ such that $\pi_{i} f=f_{i}$, which implies that $(\iota f) \iota=\iota$. Since $E$ is semisimple-directinjective envelope of $M$, we have $\iota f$ is an isomorphism. It follows that $E \cong N \oplus E(N)$ is a semisimple-direct-injective module. Thus $N=E(N)$ is injective, a contradiction.

The rest is similar.

Lemma 3.2. If $A$ is a $C 3$-module and $A \oplus E(A)$ has a $C 3$-cover, then $A$ is injective.

Proof. This similar to Lemma 3.1.

Theorem 3.3. The followings are equivalent for a ring $R$ :

(1) $R$ is an Artinian serial ring with $J(R)^{2}=0$.

(2) Every simple-direct-injective right $R$-module has a C3-cover.

(3) (a) Every semisimple-direct-injective right $R$-module has a C3-cover.

(b) The module $\oplus_{\mathfrak{J}} S_{i}$ is injective for any family of simple injective modules $\left\{S_{i}\right\}_{\mathcal{J}}$.

Proof. (1) $\Rightarrow(2)$ This is clear.

$(2) \Rightarrow(1)$ Consider the family $\left\{E_{i}\right\}_{i \in I}$ of injective right $R$-modules $E_{i}, i \in I$. By the assumption, $M=E \oplus\left(\oplus_{i \in I} E_{i}\right)$ with $E=E\left(\oplus_{i \in I} E_{i}\right)$ has a $C$-cover, say $\alpha: C \rightarrow M$. Let $E_{i_{0}}:=E$ and $\iota_{i}: E_{i} \rightarrow M$ be the inclusion maps for all $i \in I \cup\left\{i_{0}\right\}$. Since $E_{i}$ is injective (hence simple-direct-injective), there exists a linear map $\beta_{i}: E_{i} \rightarrow C$ such that $\alpha \beta_{i}=\iota_{i}$. Hence $i d=\oplus \iota_{i}=\alpha\left(\oplus \beta_{i}\right)$ which implies that $M$ is a direct summand of $C$. So $M$ is a $C 3$-module. By [5, Lemma 3.2], $\oplus_{i \in I} E_{i}$ is injective. Thus $R$ is right Noetherian.

We next prove that $R$ is right semi-Artinian. Without loss of generality, we can assume that $M$ is a non-zero indecomposable right $R$-module with $\operatorname{Soc}(M)=0$ (since $R$ is right Noetherian). Then $M$ is a $C 3$-module. Since $\operatorname{Soc}(M \oplus E(M))=0$, we get $M \oplus E(M)$ is a simple-direct-injective module. By the assumption, $M \oplus E(M)$ has a $C 3$-cover. By Lemma 3.2, $M$ is injective. Hence $M$ is uniform and every submodule of $M$ is $C 3$. Let $N$ be a non-zero arbitrary submodule of $M$. By the same argument, we have $N$ is injective. So, $N$ is a direct summand of $M$. This shows that $M$ is a semisimple module, a contradiction. Thus, every non-zero indecomposable right $R$-module has non-zero socle. It follows that $R$ is right semi-Artinian and hence $R$ is right Artinian.

By the same technique of $[5$, Theorem $3.4(1) \Rightarrow(3)]$, we can obtain that every right $R$ module is a direct sum of a semisimple module and a family of injective uniserial modules of length 2. Thus $R$ is an Artinian serial ring with $J(R)^{2}=0$.

$(1) \Leftrightarrow(3)$ This is similar to $(1) \Leftrightarrow(2)$.

Now, we can prove that the classes of semisimple-direct-injective modules over a ring $R$ provide for sdi-envelopes and sdi-covers only if $R$ is a right Noetherian right V-ring:

Theorem 3.4. The following conditions are equivalent:

(1) $R$ is a right Noetherian right V-ring.

(2) Every right $R$-module has an sdi-cover.

(3) Direct sums of semisimple-direct-injective modules have sdi-covers.

(4) Every right $R$-module has an sdi-envelope.

(5) Direct sums of semisimple-direct-injective modules has an sdi-envelope.

Proof. (1) $\Rightarrow(2),(3)$ are obvious.

$(2) \Rightarrow(1)$ For any semisimple right $R$-module $S$, then by the assumption, $M=S \oplus E(S)$ has an sdi-cover, say $\alpha: C \rightarrow M$. Let $\iota_{1}: S \rightarrow M$ and $\iota_{2}: E(S) \rightarrow M$ be the inclusion maps for all $i=1,2$. Note that $S$ and $E(S)$ are semisimple-direct-injective modules, and there are homomorphisms $\beta_{1}: S \rightarrow C, \beta_{2}: E(S) \rightarrow C$ such that $\alpha \beta_{i}=\iota_{i}$. Clearly, $i d_{M}=\iota_{1} \oplus \iota_{2}=\alpha\left(\iota_{1} \oplus \iota_{2}\right)$. This shows that $M$ is isomorphic to a direct summand of $C$, which implies that $M$ is a semisimple-direct-injective module. Hence $S$ is injective.

$(3) \Rightarrow(1)$ is similar to $(2) \Rightarrow(1)$.

$(4) \Rightarrow(1)$ Let $N$ be an arbitrary semisimple module. Assume that $\iota: M=N \oplus E(N) \rightarrow$ $E$ is the sdi-envelope, where $E$ is a simple-direct-injective module. Since $N$ and $E(N)$ 
are semisimple-direct-injective modules, there exist $f_{1}: E \rightarrow N, f_{2}: E \rightarrow E(N)$ such that $f_{i} \iota=\pi_{i}$, where $\pi_{1}: M \rightarrow N_{i}$ and $\pi_{2}: M \rightarrow E(N)$ are the projections. There exists $\phi: E \rightarrow M$ such that $\pi_{i} \phi=f_{i}$ for all $i=1,2$. It follows that $\phi \iota=i d_{M}$, and so the monomorphism $\iota$ splits. Thus $N \oplus E(N)$ is isomorphic to a direct summand of $E$. It follows that $N \oplus E(N)$ is also a semisimple-direct-injective module. Hence $N$ is injective.

$(5) \Rightarrow(1)$ is similar to $(4) \Rightarrow(1)$.

Acknowledgment. We would like to thank the referee for carefully reading the paper. The suggestions of the referee have improved the presentation of this paper. The work of A.N. Abyzov and D.N. Tapkin was performed as part of the development program of the Scientific and Educational Mathematical Center of the Volga Federal District (Agreement No. 075-02-2020-1478).

\section{References}

[1] I. Amin, Y. Ibrahim and M .F. Yousif, C3-modules, Algebra Colloq. 22 (4), 655-670, 2015.

[2] I. Amin, M.F. Yousif and N. Zeyada, Soc-injective rings and modules, Commun. Algebra, 33, 4229-4250, 2005.

[3] F.W. Anderson and K. R. Fuller, Rings and Categories of Modules, Springer-Verlag, New York, 1974.

[4] K.I. Beidar and W. F. Ke, On essential extensions of direct sums of injective modules, Archiv. Math. 78, 120-123, 2002.

[5] V. Camillo, Y. Ibrahim, M. Yousif and Y. Zhou, Simple-direct-injective modules, J. Algebra 420, 39-53, 2014.

[6] N.V. Dung, D.V. Huynh, P.F. Smith and R. Wisbauer, Extending modules, Pitman Research Notes in Math. 313, Longman, Harlow, New York, 1994.

[7] E.E. Enochs, Injective and flat covers, envelopes and resolvents, Israel J. Math. 39, 189-209, 1981.

[8] J.W. Fisher, Von Neumann regular rings versus V-rings, in: Lect. Notes Pure Appl. Math. 7, 101-119, Dekker, New York, 1974.

[9] K.R. Goodearl, Von Neumann Regular Rings, Krieger Publishing Company, Malabar, Florida, 1991.

[10] J. Hausen, Modules with the summand intersection property, Commun. Alg. 17, 135$148,1989$.

[11] Y. Ibrahim, M.T. Koşan, M. Yousif and T.C. Quynh, Simple-direct-projective modules, Commun. Algebra, 44 (12), 5163-5178, 2014.

[12] Y. Ibrahim, T.C. Quynh and M. Yousif, Simple-direct-modules, Commun. Algebra, 45 (8), 3643-3652, 2017.

[13] S.H. Mohammed and B.J. Müller, Continous and Discrete Modules, London Math. Soc. LN 147, Cambridge Univ. Press., 1990.

[14] W.K. Nicholson and M.F. Yousif, Quasi-Frobenius Rings, Cambridge Univ. Press., 2003.

[15] T.C. Quynh and M.T. Koşan, On annihilators and quasi-Frobenius rings, submitted.

[16] S. Sahinkaya and J. Trlifaj, Generalized injectivity and approximations, Commun. Algebra, 44 (9), 4047-4055, 2014.

[17] G.V. Wilson, Modules with the Direct Summand Intersection Property, Comm. Alg. 14, 21-38, 1986.

[18] R. Wisbauer, Foundations of Module and Ring Theory, Gordon and Breach, Reading, 1991. 